\title{
Neuer Geschäftsführer der Deutschen Röntgengesellschaft
}

\author{
Interview mit Herrn Dr. med. Stefan Lohwasser, dem neuen \\ Geschäftsführer der Deutschen Röntgengesellschaft - \\ Gesellschaft für Medizinische Radiologie e.V.
}

\section{? Herr Dr. Lohwasser, Sie sind seit Februar neuer Geschäftsführer der Deutschen Röntgengesellschaft und damit „ein Oberfranke in Berlin“. Haben Sie sich schon eingelebt? Lohwasser: Ja, das habe ich. Aber Berlin ist natürlich nicht neu für mich. Auch in mei- nen früheren Tätigkeiten bei der Deut- schen Forschungsgemeinschaft und beim Wissenschaftsrat war ich häufig in Berlin. Es haben in Berlin viele Sitzungen, Ge- spräche und Besprechungen stattgefun- den, insofern, ist mir die Stadt vertraut und das Einleben fällt mir deshalb relativ einfach.}

\section{? Sie sind selbst Mediziner. In wel- chem Fachgebiet und wie lange sind Sie als praktizierender Arzt tä- tig gewesen?}

Lohwasser: Ich habe in Erlangen Medizin studiert und war danach mehrere Jahre am Universitätsklinikum Regensburg in der Abteilung für Hämatologie und Onkologie beschäftigt. Nach dieser Krankenversorgungszeit bin ich in die Grundlagenforschung gegangen und war mehrere Jahre in Vancouver, Kanada, tätig. Danach bin ich ins Wissenschaftsmanagement gewechselt. Zuerst war ich bei der Deutschen Forschungsgemeinschaft und dann zum Schluss in der Politikberatung, Wissenschafts- und Gesundheitspolitik beim Wissenschaftsrat in Köln.

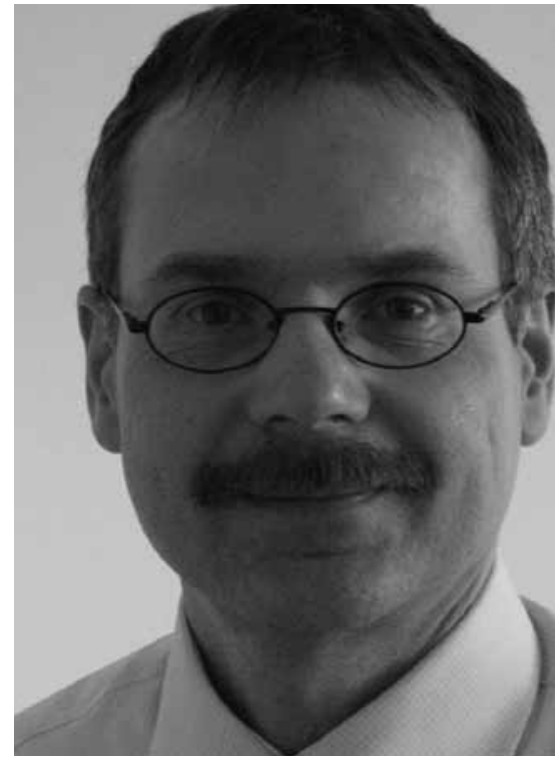

\section{?} Sie sich Fortbildung und Nachwuchsförderung auf die Fahnen geschrieben. Wie sehen Ihre konkreten Vorstellungen diesbezüglich aus?

Lohwasser: Die Deutsche Röntgengesellschaft hat ja schon viele vorbildliche Strukturen in diesem Bereich aufgebaut. Als eine der ersten Fachgesellschaften hat sie 1998 eine Akademie für Fortbildung gegründet - war also sehr früh in diesem Bereich tätig. In den vergangenen Monaten haben wir diesen Fortbildungsbereich auch ins Internet gehoben und haben die
„Mir machen der Austausch und die Gespräche mit vielen verschiedenen Menschen am meisten Spaß“,

sagt Dr. med. Stefan Lohwasser mit Blick auf seine neue Aufgabe bei der Deutschen Röntgengesellschaft.

Lohwasser, Jahrgang 1968, ist gebürtiger Oberfranke und tritt die Nachfolge von Bernhard

Lewerich an.

Akademie jetzt online, wo etwa in 2-wöchigen Abständen Fortbildungsveranstaltungen angehört und angesehen werden können.

Im Bereich der Nachwuchsförderung sind wir im Forschungsbereich mit einer Plattform tätig, die wir „Forscher für die $\mathrm{Zu}$ kunft“ nennen. Dadurch versuchen wir junge Forscher aus der Radiologie zusammenzubringen, zu vernetzten, sie letztendlich durch Workshops fit für die Forschung zu machen und auch nach diesen Workshops den Kontakt aufrechtzuerhalten. Natürlich auch in der Hoffnung, dass daraus neue Forschungsprojekte entstehen. 
Auch im Bereich der Studierenden sind wir mit einer Plattform, die „Hellste Köpfe" heißt, aktiv. Wir versuchen Studierende anzusprechen und sie mit einem Paten- und Stipendienprogramm zum Röntgenkongress zu bringen. Hier beschäftigen wir uns auch zum ersten Mal mit sozialem Web und versuchen auch in diesen Bereich hineinzugehen.

Die soeben genannten Maßnahmen bestehen bereits, aber manche sind noch relativ neu. Jetzt ist natürlich die Frage, welche Maßnahmen erfolgreich sind, welche weiterentwickelt und welche zusätzlich angegangen werden müssen.

\section{? Bis vor Kurzem waren Sie bei} der Deutschen Forschungsgemeinschaft beschäftigt. Haben Sie deshalb die Förderung der Radiologie im Wissenschaftsbetrieb in Ihren Fokus gerückt? Was ist für Sie dabei der wichtigste Punkt?

Lohwasser: Meine eigene Sichtweise ist natürlich auch geprägt durch meinen eigenen Werdegang in der Krankenversorgung, aber auch in der aktiven Forschung, im Forschungsmanagement und auch in der Politikberatung. Für jede medizinische Fachdisziplin ist es von zentraler Bedeutung, dass sie sich auch akademisch im Forschungsbereich weiterentwickelt, weil nur so, kann sie im Konzert oder auch im Wettbewerb der verschiedenen Disziplinen auf lange Sicht überleben. Deshalb ist die Forschung ein wichtiger Bestandteil der Weiterentwicklung eines Faches, und das ist natürlich die vornehmste Aufgabe einer wissenschaftlichen medizinischen Fachgesellschaft.

\section{?} hen aus Ihrer Sicht bei der Deut-
schen Röntgengesellschaft als Fachgesellschaft im IMittelpunkt?

Lohwasser: Da würde ich hauptsächlich 2 Punkte benennen. Derzeit wird sehr intensiv in der Medizintechnik über KostenNutzen-Analysen und über Qualitätsmaßnahmen diskutiert. Die Diskussion ist diesbezüglich sehr geprägt von den Erfahrungen, die im Pharmabereich gemacht wurden. Diese Regularien und gesetzlichen Regeln sind aus meiner Sicht aber nicht 1 zu 1 auf den medizinischen-technischen Bereich übertragbar. Hier ist es wichtig, dass die Radiologie ein Angebot macht und aufzeigt, welche Wege in diesem Bereich gegangen werden können.
Dabei ist auch von zentraler Bedeutung, dass die Studienfähigkeit der Radiologie verbessert wird. Deshalb versuchen wir über unsere bereits erwähnte Plattform „Forscher für die Zukunft“ - die wir thematisch sehr stark auf translationale Forschung ausgerichtet haben - klinische Studien innerhalb der Radiologie anzuregen.

Der andere Bereich, der für mich sehr wichtig ist, ist die Verbesserung der Zusammenarbeit mit den anderen Bild- und Strahlenfächern. Wir kooperieren unter anderem schon mit der Deutschen Gesellschaft für Nuklearmedizin und der Deutschen Gesellschaft für Radioonkologie in einer Image Campagne, die wir „Medizin mit Durchblick“ nennen. Aber ich denke, dass die inhaltliche Kooperation in diesem Bereich noch verbessert werden kann und es auch sehr wichtig ist, zwischen diesen Fachgesellschaften Vertrauen zu schaffen.

\section{? Als Geschäftsführer der Deut-} schen Röntgengesellschaft wird Sie ein buntes Portfolio an Aufgaben erwarten. Worauf freuen Sie sich persönlich am meisten?

Lohwasser: Wie schon in meinen Vortätigkeiten, machen mir der Austausch und die Gespräche mit vielen verschiedenen Menschen am meisten Spaß. Ich habe jetzt schon das Gefühl, von der Deutschen Röntgengesellschaft gut aufgenommen worden zu sein. Und ich freue mich natürlich auf weitere Begegnungen, angefangen von Studierenden, über Nachwuchsforscher, junge Ärztinnen und Ärzte in der Weiterbildung, bis hin zu den arrivierten Radiologen. Das sind die Dinge, auf die ich mich am meisten freue.

Das Interview führte Dr. Claudia GampeBraig, Stuttgart. 